\title{
A New Travel Time Prediction Method for Intelligent Transportation System
}

\author{
Lutfun Nahar ${ }^{1}$, Zinnia Sultana ${ }^{2}$ \\ ${ }^{\text {I }(C o m p u t e r ~ S c i e n c e ~ \& ~ E n g i n e e r i n g, ~ I n t e r n a t i o n a l ~ I s l a m i c ~ U n i v e r s i t y ~ C h i t t a g o n g, ~ B a n g l a d e s h) ~}$ \\ ${ }^{2}$ (Computer Science \& Engineering, International Islamic University Chittagong, Bangladesh)
}

\begin{abstract}
Travel time prediction is an indispensable for numerous intelligent transportation systems (ITS) including advanced traveler information systems. The main purpose of this research is to develop a dynamic travel time prediction model for road networks. In this study we proposed a new method to predict travel times using Artificial Neural Network model because artificial neural network has exhibited high accuracy and speed when applied to large databases. In addition, we compare the proposed method with such prediction methods as link-based prediction model and time varying coefficient linear regression model. It is shown from our experiment that ANN predictor can reduce mean absolute relative error significantly rather than the other predictors. We illustrate that ANN is suitable and performs well for traffic data analysis.
\end{abstract}

Keywords: ATIS (Advanced Travellers Information Systems), Chain Average (CA), Intelligent Transportation System (ITS), K-means Clustering, Nä̈ve Bayesian (NBC), Successive Moving Average (SMA)

\section{Introduction}

Recently, accurate estimation of travel times has been central for traffic data analysis to various Advanced Travelers Information System (ATIS) and ITS applications such as trip planning, vehicular navigation systems and dynamic route guidance systems. Moreover, Travel time prediction is also becoming increasingly important with the development of ATIS [1]. In addition, Travel time forecasting provides information that helps travelers to decide whether they should change their routes, travel mode, starting time or even cancel their trip [2]. So, the reliable and accurate travel time prediction on road network plays an important role in any kind of dynamic route guidance systems to fulfill the users' desires. On top of that, the importance of travel time information is also indispensable to find the fastest path (i.e. shortest path according to travel time) that connects the origin and destination. Besides, accurate travel time information also helps delivery industries to progress their service quality by delivering on time.

Travel time prediction is based on vehicle speed, traffic flow and occupancy which are extremely sensitive to external event like weather condition and traffic incident [3]. Addressing the uncertainty on the road network is also a crucial issue in the research domain. Prediction on uncertain situation is very complex, so it is important to reach optimal accuracy. Yet, the structure of the traffic flow of a specific road network fluctuates based on daily, weekly and occasional events. For example, the traffic condition of weekend may differ from that of weekday. So, time-varying feature of traffic flow is one of the major issues to estimate accurate travel time[12]. In this study, we focus a new method that is able to predict travel time reliably and accurately. In this research we have tried to combine the advantages of our previous methods namely NBC [12],SMA and CA[13] by eliminating the shortcomings of those methods. Proposed ANN method is able to address the arbitrary route on road networks that is given by user. Furthermore proposed method flushes a functional relationship between traffic data as input variables and predicted travel time as the output variables.

According to the experimental result, our method exhibits satisfactory performance in terms of prediction accuracy. At the same time, the result is considered to be superior rather than other prediction methods like NBC, switching method,SMA and CA.

\section{Literature Review And Motivation}

Travel time prediction has emerged as an active and intense research area nowadays. In the literature, there are a large number of researches that can deal with accurate prediction of travel time on road networks. In the following section, a wide-ranging literature review on the topic of travel time prediction is presented .Travel time prediction methods are broadly categorized in two parts, named as path-based estimation and link-base estimation. Most studies are focused on path travel time estimation, it is generally assumed that path travel time is the travel time between any two points in a road network.

In this section ,a historical background on the topic of travel time prediction is discussed briefly. For instance, Park et al.[5],[6]proposed Artificial Neural Network (ANN) models for forecasting freeway corridor travel time rather than link travel time. One model used a Kohonen Self Organizing Feature Map (SOFM) while the other utilized a fuzzy c-means clustering technique for traffic pattern classification. Lint et al[7],[8] proposed 
a state space neural network based approach to provide robust travel time predictions in the presence of gaps in traffic data.

Kwon et al [9] focused on linear regression method. They used an approach to estimate travel time on freeways derived from flow and occupancy data from single loop detectors and historical travel time information. Their proposed predictor was a linear combination of the current and historical information. Zhang et al proposed a method to predict freeway travel times using a linear model in which the coefficients vary as smooth functions of the departure time.

A linear predictor consisting of a linear combination of the current times and the historical means of the travel times was proposed by Rice et al[10]. They presented a method to predict the time that would be needed to traverse a given time in the future.

Most recent research in this field is proposed by Erick et al[11] . They investigated a switching model which was consisted of two linear predictors. Beside this, they have shown that there is a point in future time where the linear predictor is no longer better than the historical mean. That means this point is varied according to day and time for a given roadway.

On the other hand, a few researches have investigated the use of link travel time to model travel time prediction. It is assumed that link travel time prediction is the addition of the travel times on its consisting links for a particular route. Chen et al conducted a study that focused on link travel time prediction. Their study compared the prediction accuracy under direct measurements of path-based travel time versus link-based travel times. For path-based method, probe vehicle's passing is only recorded at the beginning and the end of the path. The average probe travel time is used as the real-time observation of travel time at each time period. In linkbased method, record travel times on desired links for those probes entering the links and get the average probe travel time for each link. Final travel time is calculated by adding travel times on all consisting links.

In the past research, we have observed that prediction algorithms are trained on particular route regardless of other routes in road networks. Kwon et al[9]. Employed their approach for arbitrary travel routes. In their approach, at first they partition the freeway into short segments and observe future travel time on every segment. On the other hand, this approach takes more storage and computation time due to two-step computation.

In previous an efficient method for predicting travel time by using NBC was proposed by Lee et al [12] which had also been scalable to road networks with arbitrary travel routes. The main idea of NBC was that it would give probable velocity level for any road segment based on historical traffic data. It was shown from experiments that NBC could reduce MARE significantly rather than the other predictors.

In previous research, two completely new methods namely SMA and CA proposed by Lee et al [13] that were based on moving average. In that research,it eliminate the drawbacks of conventional moving average approach such as unwanted fluctuation in data set. These methods were also scalable to large network with arbitrary travel routes. Moreover, both methods were less expensive in terms of computational time.

Consequently, it was revealed that these proposed methods can reduce error significantly, compared with existing methods [13].Travel time prediction forms an integral part of any ATIS. The grouping style of whole day is efficiently and effectively done by NBC. But a significant problem will arise when we calculate velocity level for a particular route. Moreover, this method emphasize on those data whose probabilities are higher i.e. it does not concern with all data. Although NBC is capable to predict more accurately ordinary, usually it doesn't give significant result in the uncertain situation. Nevertheless, we have to compute velocity class prior probability and velocity class posterior probability.

So, it takes more computational time. On the other hand, SMA and CA compute all data which are not based on probability theory. Though they provide an almost accurate travel time, they are also failed to find out uncertain data from the available traffic data.

Another prediction method called Modified K-means clustering [MKC]was proposed by Hyun-jo Lee[14] for discovering hidden knowledge that can be applied in the large historical traffic data set .To address the uncertain situation and predict the travel time more accurately. This eliminate the shortcomings of traditional K-means Clustering. But here is some problem like dissimilarity in the measure unit .It sum the difference between two historical data in each of the three dimentions. Different units were used for each dimention such as difference in travel times were measured in minutes,difference in velocity were measured in $\mathrm{km} / \mathrm{h}$.

There are various knowledge based techniques that can be used in the traffic data. Artificial Neural Network is one of the leading tools for discovering hidden knowledge that can be applied in the large historical traffic data set. To address the uncertain situation and predict the travel time more accurately, we propose ANN method. The key challenges of this research are to reduce prediction error as well as to predict the uncertain situation. At the same time, proposed method can also be scalable to large network with arbitrary travel routes. To motivate the presentation of proposed method, the following sections will explore complete scenario of our proposed method. 


\section{Proposed Travel Time Prediction Method}

In this section, we propose a new method for predicting travel time from historical traffic data using Artificial Neural Network .One type of network sees the nodes as 'artificial neurons'. These are called artificial neural networks (ANNs). Initially, an origin with start time and destination is initialized by user. A route may consist of several road segments from origin to destination. First of all, we apply our ANN method on the data set of the first road segment to calculate the end time of first road segment which in turn becomes the start time of the next road segment. Finally, applying successive repetition approximate travel time from origin to destination can be measured.Again, the road environment of the same road network for running vehicles on the different time periods of a day is different. So, for our convenience, the whole day time is separated into several groups according to the time as shown in Table 1:

Table 1. Time Group definition

\begin{tabular}{|l|l|}
\hline Start_time_range & Time_Group \\
\hline $06: 01-10: 00$ & 1 \\
\hline $10: 01-11: 00$ & 2 \\
\hline $11: 01-12: 00$ & 3 \\
\hline $12: 01-14: 00$ & 4 \\
\hline $14: 01-16: 00$ & 5 \\
\hline $16: 01-18: 00$ & 6 \\
\hline $18: 01-22: 00$ & 7 \\
\hline $22: 01-00: 00$ & 8 \\
\hline $00: 01-06: 00$ & 9 \\
\hline
\end{tabular}

Table 2. Sample historical traffic data

\begin{tabular}{|c|c|c|c|c|c|c|}
\hline Vehicle_ID & Road_ID & Time_group & Start_time & End_time & $\begin{array}{l}\text { Travel_time } \\
(\mathrm{sec})\end{array}$ & $\begin{array}{l}\text { Velocity } \\
(\mathrm{m} / \mathrm{s})\end{array}$ \\
\hline 1 & 1 & 6 & $16: 50: 07$ & $16: 50: 10$ & 3 & 15.315333 \\
\hline 2 & 1 & 6 & $17: 20: 28$ & $17: 20: 36$ & 8 & 7.227000 \\
\hline 3 & 1 & 6 & $17: 43: 02$ & $17: 43: 14$ & 12 & 10.115251 \\
\hline 4 & 1 & 6 & $16: 02: 12$ & $16: 02: 15$ & 3 & 15.315333 \\
\hline 5 & 1 & 6 & $16: 16: 20$ & $16: 16: 28$ & 8 & 7.227000 \\
\hline 6 & 1 & 6 & $16: 05: 20$ & $16: 05: 32$ & 12 & 10.115251 \\
\hline 7 & 1 & 6 & $17: 03: 21$ & $17: 03: 29$ & 8 & 7.227000 \\
\hline 8 & 1 & 6 & $17: 18: 12$ & $17: 18: 26$ & 14 & 5.120286 \\
\hline 9 & 1 & 6 & $17: 35: 07$ & $17: 35: 12$ & 5 & 12.365600 \\
\hline 10 & 1 & 6 & 16:09:18 & 16:09:30 & 12 & 10.115251 \\
\hline
\end{tabular}

For example: if a vehicle starts from any road segment between 16:01 and 18:00,its Time_group will be 6. Table 2 illustrates the sample snapshot of historical traffic data for any road segment. Each record of the table contains seven attributes. The value of Time_group is calculated from the Start_time. Travel_time is the difference from End_time to Start_time. Dividing length of road segment by Travel_time, Velocity is measured. To calculate approximate travel time for any road segment, we introduce ANN method in the following section with appropriate example.

1.1 Explanation of Modified Feed Forward Neural Network Method:

PROCEDURE

Step1:

1.At first convert the travel time from sec to min.

2.convert the velocity from $\mathrm{m} / \mathrm{s}$ to $\mathrm{m} / \mathrm{min}$.

Step 2:

Count the frequency for each travel time by counting the repetition of that travel time in different records.

Step 3:

Classify each unique travel time for input.

Step 4: 
Use each unique travel time as node for Feed Forward Neural Network in the input layer.

Step 5:

For each node give the value of edge which is weight.

Step 6:

In the hidden layer we use two node.one's edge value is velocity and the other' edge value is frequency.

Suppose,the name of two node is I and J.

Step 7:Now we find the value of I,

$\mathrm{I}=$ weight*travel time

$=$ velocity*travel time

$=\mathrm{vt}$

And the value of $\mathrm{J}$,

$\mathrm{J}=$ weight*travel time

$=$ frequency*travel time

$=\mathrm{ft}$

Step8:

Find predicted trvel time from step 7

In the I node:

$\mathrm{T}=\mathrm{vt} / \mathrm{total} \mathrm{v}$.

Again In the J node:

$\mathrm{T}=\mathrm{ft} / \mathrm{total} \mathrm{f}$.

Step9:

Pass the Ith node and the Jth node travel time into activation function, where we use logistic sigmoid function as activation function.

I th node:

Predicted travel time $\mathrm{P}(\mathrm{I})=1 / 1+\mathrm{e}^{\wedge}-\mathrm{t}$.

J th node:

Predicted Travel time $\mathrm{P}(\mathrm{J})=1 / 1+\mathrm{e}^{\wedge}$-t.

Step 10:

Now we pass the travel time find from step9 as input to the output layer.Where edge weight is 1.00 (constant).

Multiply the travel time with edge weight and apply the logistic sigmoid function .

Step11:

Finally we get the desire travel time from the output layer,P(o).

Step12:

Find the difference between the Ith node travel time and the Jth node travel time find from step9 .Then again find the difference among the Ith node travel time ,Jth node travel time and final travel time find from output layer at step11.

Step13:

$\mathrm{P}(\mathrm{t})=[|\mathrm{P}(\mathrm{o})-\mathrm{P}(\mathrm{I})|+|\mathrm{p}(\mathrm{o})-\mathrm{p}(\mathrm{J})|] / 2$

$P(m)=|p(I)-p(j)|$

Average travel time $=[\mathrm{p}(\mathrm{t})+\mathrm{p}(\mathrm{m})] / 2$

Step14:

Finally convert the desire travel time in sec by multiplying by 60.Explanation of Modified Feed Forward Neural Network

Method with example:

Considering the simple historical traffic data of table 2 that containing data Road_id=1

And Time_group $=6$ the step of FFNN procedure are explained below:

Step 1: : There are 10 records in Table 2 where Road_id and Time_group are common. First step of FFNN reveals to convert the travel_time from $\min$ to $\mathrm{sec}$ and the velocity from $\mathrm{m} / \mathrm{s}$ to $\mathrm{m} / \mathrm{min}$.

Step 2: There are 10 records in Table 2 where Road_id and Time_group are common.

Second step of FFNN reveals to find the frequency of each distinct travel time. If we observe Table 2, then we find that the frequency of Travel_time 3 is two (2) because the number of repetition of Travel_time 3 in different records is two. Similarly, frequencies of Travel_time 8,12,14, and 5 are 3,3,1, and 1 respectively.

\begin{tabular}{|l|l|l|}
\hline Travel_time(min & Frequency & Velocity $(\mathrm{m} / \mathrm{min})$ \\
\hline 0.05 & 2 & 918.91998 \\
\hline 0.134 & 3 & 433.62 \\
\hline 0.2 & 3 & 606.91506 \\
\hline 0.234 & 1 & 307.21716 \\
\hline 0.0834 & 1 & 741.936 \\
\hline
\end{tabular}


The table are shown here:

Step 3:

Classify each unique travel time for input.

Input:

$0.05,0.134,0.2,0.234,0.0834$

Step 4,5,6,7:

Now we find the value of I,

I=weight*travel time

$=$ velocity*travel time

$=\mathrm{vt}$

$\mathrm{I}=0.05 * 918.91998+0.134 * 433.62+0.2 * 606.91506+0.234 * 307.21716+0.0834 * 741.936$

$=359.2003688$

And the value of $\mathrm{J}$,

$\mathrm{J}=$ weight $*$ travel time

$=$ frequency*travel time

$=\mathrm{ft}$

$\mathrm{J}=0.05 * 2+0.134 * 3+0.2 * 3+0.234 * 1+0.0834 * 1$

$=1.4194$

Step8:

Find predicted travel time from step 7

In the I node:

$\mathrm{T}=\mathrm{vt} /$ total $\mathrm{v}$.

$\mathrm{T}=359.2003688 / 3008.6082$

$=0.1193908761$

Again In the $\mathrm{J}$ node:

$\mathrm{T}=\mathrm{ft} /$ total $\mathrm{f}$.

$=1.4194 / 10$

$=0.14194$

Step9:

Pass the Ith node and the Jth node travel time into activation function, where we use logistic sigmoid function as activation function.

I th node:

Predicted travel time $\mathrm{P}(\mathrm{I})=1 / 1+\mathrm{e}^{\wedge}-\mathrm{t}$.

$\mathrm{P}(\mathrm{I})=1 / 1+\mathrm{e}^{\wedge}-0.1193908761=0.5298$

$\mathrm{J}$ th node:Predicted Travel time $\mathrm{P}(\mathrm{J})=1 / 1+\mathrm{e}^{\wedge}-\mathrm{t}$.

$\mathrm{P}(\mathrm{J})=1 / 1+\mathrm{e}^{\wedge}-0.14194=0.5354$

Step 10,11:

Pass the travel time find from step9 as input to the output layer. Where edge weight is 1.00 (constant). Multiply the travel time with edge weight and apply the logistic sigmoid function, we get the desire travel time from the output layer, $\mathrm{P}(\mathrm{o})$.

$\mathrm{o}=0.5298 * 1.00+0.5354 * 1.00=1.06523$

$\mathrm{p}(\mathrm{o})=1 / 1+\mathrm{e}^{\wedge}-\mathrm{o}=1 / 1+\mathrm{e}^{\wedge}-1.0652=0.7436$

Step12 and13:

$\mathrm{P}(\mathrm{t})=[|\mathrm{P}(\mathrm{o})-\mathrm{P}(\mathrm{I})|+|\mathrm{p}(\mathrm{o})-\mathrm{p}(\mathrm{J})|] / 2$ $=[|0.7436-0.5298|+|0.7436-0.5354|] / 2=0.211$

$\mathrm{P}(\mathrm{m})=|\mathrm{p}(\mathrm{I})-\mathrm{p}(\mathrm{j})|$ $=|0.5298-0.5354|=0.0056$

Average travel time $=[\mathrm{p}(\mathrm{t})+\mathrm{p}(\mathrm{m})] / 2=[0.211+0.0056] / 2=0.1083 \mathrm{~min}$

Step14:

Finally convert the desire travel time in sec by multiplying by 60 .

Average time $=0.1083 * 6=6.498 \mathrm{sec}$

So the final approximation travel time $=6.5 \mathrm{sec}$

IV. Simulations Results

A real data set that used was collected by Pusan National University (PNU) generator [12] to measure the performance of different predictors. This generator is based on real traffic situation in Pusan city .South Korea. Global Positioning System (GPS) sensor is used to collect real traffic delay for building this wellorganized PNU generator. Traffic pattern of Pusan city was extracted from this data. According to this traffic 
pattern, generator simulates and generates trajectory data which is almost same as real data. By using this generator, 167,669 trajectories are generated.

Every trajectory may be composed of several road segments. This data organization format sufficiently reflects real traffic situations. For computing easily and efficiently and accurate evaluation of performance of the algorithms, data is divided into two categories, namely training data and test data sets. 365 days traffic data are used as training data set and 30 days traffic data are used as testing data set. Testing data sets are chronologically after 365 days data used for training data from 365 training days are used for fitting the model. However, 30 days test data are used to measure prediction performance for all methods.

\section{Comparison And Prediction Accuracy}

MARE are used to compare the accuracy among all prediction methods. MARE is the simplest and well-known method for measuring overall error in travel time prediction. MARE measures the magnitude of the relative error over the desired time range. The MARE is measured by the following form

$$
\text { MARE }=\frac{1}{N} \sum_{i=1}^{N} \frac{\left|x(t)-x^{*}(t)\right|}{x(t)}
$$

Where $\mathrm{X}(\mathrm{t})=$ Observation value

$\mathrm{X}^{*}(\mathrm{t})=$ predicted value

$\mathrm{N}=$ Number of Sample

VI. Performance Analysis

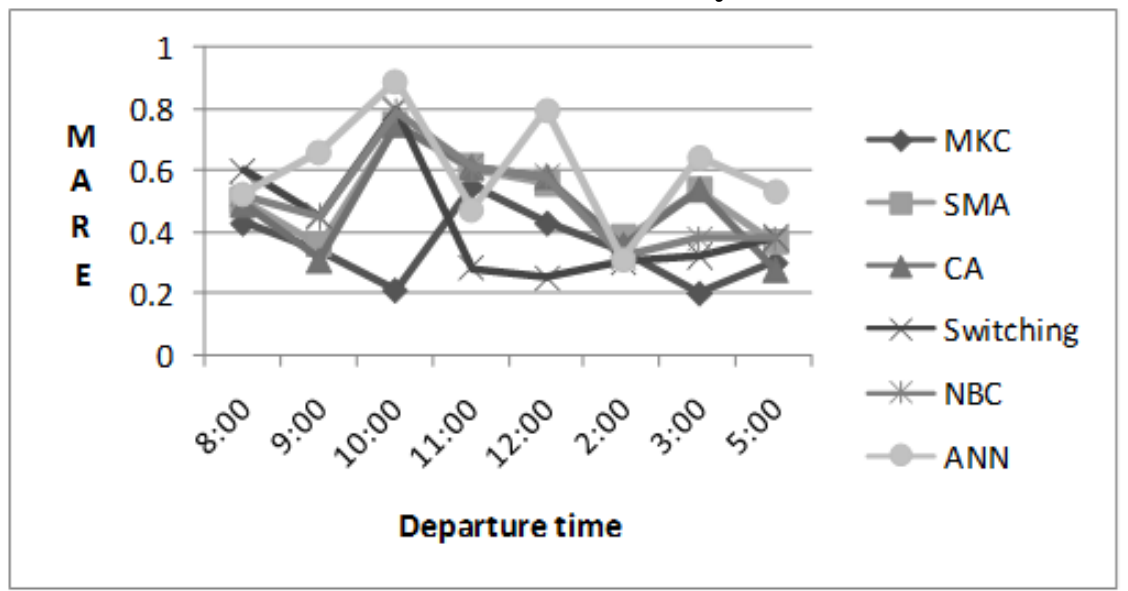

Fig :1

The line chart shown in Fig:1 illustrates relative performance of all travel time predictors.From the overall point of view, proposed method performs much better than NBC, SMA ,CA,Switching and MKC method in many cases, it is shown that five test cases exhibit errors less than 0.50. At 09.00 AM, 11.00 PM and 2.00 PM our method ANN predicted more accurately than others and datasets of those period included uncertain data.

\section{Conclusion And Future Work}

Though there are many studies on predicting travel time in a road network, there exists little research on travel time prediction by considering arbitrary routes.

In our study, the paper focuses on travel time prediction in road network for ATIS. we have developed a methods for predicting travel time by using real traffic data from PNU trajectory generator. We see that, simulation results suggest that proposed methods provide a more precise prediction in most test cases.

Future work will include an analysis of the relationship between the length of roadways and accuracy of the prediction. Beside this, analysis of travel time prediction will be extended with respect to real field data.ANN method is able to predict in uncertain situations more accurately comparing with other methods. Moreover performance analysis portion of this research reveals that our proposed method outperforms other methods in most case. 


\section{References}

[1] M. Chen and S. Chien. "Dynamic freeway travel time prediction using probe vehicle data:Link-based vs. Path-based". J. of Transportation Research Record, TRB Paper No. 01-2887, Washington, D.C. 2001

[2] C. H. Wei and Y. Lee. "Development of Freeway Travel Time Forecasting Models by Integrating Different Sources of Traffic Data". IEEE Transactions on Vehicular Technology.Vol. 56, 2007

[3] W. Chun-Hsin, W. Chia-Chen, S. Da-Chun, C, Ming-Hua and H. Jan-Ming. "Travel Time Prediction with Support Vector Regression". IEEE Intelligent Transportation Systems Conference, 2003

[4] J. Kwon and K. Petty. "A travel time prediction algorithm scalable to freeway networks with many nodes with arbitrary travel routes". Transportation Research Board 84th Annual Meeting, Washington, D.C. 2005

[5] D. Park and L. Rilett. "Forecasting multiple-period freeway link travel times using modular neural networks". J. of Transportation Research Record, vol. 1617, pp.163-170. 1998

[6] D. Park and L. Rilett. "Spectral basis neural networks for real-time travel time forecasting". J.of Transport Engineering, vol. 125(6), pp.515-523, (1999)

[7] J. W. C. V. Lint, S. P. Hoogenoorn and H. J. V. Zuylen. "Towards a Robust Framework forFreeway Travel Time Prediction: Experiments with Simple Imputation and State-Space Neural Networks". Presented at 82 Annual Meeting of the Transportation Research Board,Washington ,D.C., 2003

[8] J. W. C. V. Lint, S. P. Hoogenoorn and H. J. V. Zuylen. "Freeway Travel Time Prediction with State-Space Neural Networks: Modeling State-Space Dynamics with Recurrent Neural Networks". In Transportation Research Record: Journal of the Transportation Research Board, No. 1811, TRB, National Research Council, Washington, D.C., pp. 30-39. 2002

[9] J. Kwon, B. Coifman and P. J. Bickel. "Day-to-day travel time trends and travel time predicti-on from loop detector data". J. of Transportation Research Record, No. 1717, TRB, National Research Council, Washington, D.C., pp. 120-129. 2000

[10] J. Rice and E. Van Zwet. "A simple and effective method for predicting travel times on freeways". In: IEEE Trans. Intelligent Transport Systems, vol. 5, no. 3, pp. 200-207, 2004

[11] J. Schmitt Erick and H. Jula. "On the Limitations of Linear Models in Predicting Travel Times". In: IEEE Intelligent Transportation Systems Conference, 2007

[12] H. Lee, N. K. Chowdhury and J. Chang. "A New Travel Time Prediction Method for Intelligent Transportation System". In: International Conference on Knowledge-Based and Intelligent Information \& Engineering Systems, LNAI 5177, pp: 473-483, 2008

[13] N. K. Chowdhury, R. P. D. Nath, H. Lee and J. Chang. "Development of an Effective Travel Time Prediction Method using Modified Moving Average Approach". 13 International Conference on Knowledge-Based and Intelligent Information \& Engineering Systems. Part 1 LNAI 5711, pp: 130-138 2009 International Journal of Data Engineering (IJDE), Volume (2) : Issue (2) :73 2011 Rudra Pratap Deb Nath, Nihad Karim Chowdhury \& Masaki Aono

[14] H. Kitaoka, T. Shiga, H. Mori, E. Teramoto and T. Inoguchi. "Development of a Travel TimePrediction Method for the TOYOTA G-BOOK Telematics service". R \& D Review of TOYOTA CRDL vol. 41 no. 4,2006

[15] S. UI, I. Bajwa and M. Kuwahara, "A Travel Time Prediction Method Based on Pattern Matching Technique". In proceedings of the 21st ARRB and 11th REAAA Conference.Transport. Vermont South, Victoria 3133, ZZ N/A Australia.2003

[16] R. P. D. Nath, H. Lee, N. K. Chowdhury and J. Chang. "Modified K-means Clustering forTravel Time Prediction Based on Historical Traffic Data". 14 International Conference onKnowledge-Based and Intelligent Information \& Engineering Systems. Part 1. LNAI 6276,pp: 511-521, 2010.

[17] J. Chang, N. K. Chowdhury and H. Lee. "New travel time prediction algorithms for intelligent transportation systems". Journal of intelligent and fuzzy systems, vol.21, pp: 5-7, 2010. 